\title{
Coarse-Grained Multifractality Analysis Based on Structure Function Measurements to Discriminate Healthy from Distressed Foetuses
}

\author{
Souad Oudjemia, ${ }^{1}$ Amira Zaylaa, ${ }^{2}$ Salah Haddab, ${ }^{1}$ and Jean-Marc Girault ${ }^{2}$ \\ ${ }^{1}$ University of Mouloud Mammeri, Tizi-Ouzou, Algeria \\ ${ }^{2}$ Signal \& Imaging Group, University François Rabelais of Tours, UMR INSERM U930, PRES Loire Valley University, \\ 7 Avenue Marcel Dassault, 37200 Tours, Cedex, France
}

Correspondence should be addressed to Jean-Marc Girault; jean-marc.girault@univ-tours.fr

Received 28 June 2013; Revised 6 November 2013; Accepted 22 November 2013

Academic Editor: Catherine Marque

Copyright (C) 2013 Souad Oudjemia et al. This is an open access article distributed under the Creative Commons Attribution License, which permits unrestricted use, distribution, and reproduction in any medium, provided the original work is properly cited.

This paper proposes a combined coarse-grained multifractal method to discriminate between distressed and normal foetuses. The coarse-graining operation was performed by means of a coarse-grained procedure and the multifractal operation was based on a structure function. The proposed method was evaluated by one hundred recordings including eighty normal foetuses and twenty distressed foetuses. We found that it was possible to discriminate between distressed and normal foetuses using the Hurst exponent, singularity, and Holder spectra.

\section{Introduction}

Foetal distress is often the result of reduction in respiratory exchange between the mother and the foetus. In most cases, foetal distress is strongly related to intrauterine growth retardation [1]. Early identification of distressed status from heart rate variability is highly important since it can help the obstetrician to decide on immediate delivery by caesarean section.

The value of analysis of heart rate variability (HRV) to provide a means of diagnosis and prognosis of heart disease is now well established. HRV time series has now become the elementary basis from which most analyses and processes are operated.

Due to the nonstationary and nonlinear nature of HRV time series, many recent studies have tried to take full advantage of the nonlinear nature of heart rate variability by analysing the complexity of time series. This complexity analysis of the foetal heart rate (FHR) that has its roots in adult HRV research can be conceived of in many ways. However, it was probably the scale invariance properties observed through power law spectral density [2] that was the triggering element for several studies based on the multiscale analysis of HRV $[3,4]$. Among the overall complexity descriptors, entropy descriptors $[3,5,6]$ with fractal dimension estimators [7-10] were probably the first "nonconventional" tools used to study FHR. Certain studies even used multifractal features of FHR. The research studies by Ivanov et al. were probably the first to demonstrate multifractality in cardiac dynamics as well as in physiologic dynamics in general [11-13]. These seminal studies were then followed by researches such as [14-17], to name but a few.

The starting point of the present study was based on two approaches, the first being that of Wang et al. [15] focusing on the multifractal analysis of adult ECG signals with a coarsegraining approach initially proposed by [18].

The second approach was based on the studies by $[19,20]$ and more recently those of $[16,21,22]$ that themselves used a method based on a structure function [23] of a time series in order to extract multifractal indicators.

In response to these two kinds of research, we investigated a coarse-grained multifractal analysis of the foetal heart rate in order to discriminate healthy from distressed foetuses.

Although the present study has certain similarities to those proposed by Wang et al. [15], our study was different in two ways. First, unlike the study based on a partition function proposed by [15], our study was based on a structure function. 
The second difference was that our coarse-graining analysis was performed on the foetal heart rate, whereas that proposed by [15] was evaluated on adult electrocardiograms.

Our study aimed to improve the differentiation between normal and distressed foetuses by investigating the time scale dependency of the multifractal features of the FHR in depth. To do so we investigated the multifractal analysis originating from a structure function from a coarse-graining point of view.

To demonstrate the value of our approach, we tested the proposed method on a dataset derived from normal and distressed foetuses.

\section{Materials}

Our system comprised a personal computer and a Doppler ultrasound unit. The latter device contained three groups of four transducers and a Doppler acquisition board. The transducers exploring the foetal heart were nonfocused and monoelement. The transducers placed on the mother's abdomen were circular in shape, with a diameter of $13.5 \mathrm{~mm}$ and an acoustic power of $1 \mathrm{~mW} / \mathrm{cm}^{2}$. Each transducer transmitted a sinusoidal pulse at $2.25 \mathrm{MHz}$ with a pulse repetition frequency of $1 \mathrm{kHz}$. The wave was propagated through the mother's abdomen towards the foetal heart.

The backscattered signal was converted into an electrical signal and amplified to compensate for the attenuation of $1 \mathrm{~dB} / \mathrm{cm} / \mathrm{MHz}$. The signal was then demodulated in phase (I) and quadrature $(\mathrm{Q})$.

The Doppler signals were acquired at CHRU "Bretonneau" Tours, France. The consent of each patient was obtained and the study was approved by the Ethics Committee of the Clinical Investigation Centre for Innovative Technology of Tours (CIC-IT 806 CHRU of Tours). All patients were over eighteen years of age and pregnancies were single. One hundred examinations (eighty normal foetuses and twenty distressed foetuses) were recorded in this study. Gestational ages of foetuses ranged from 25 to 39 weeks were monitored for 30 minutes. FHR was evaluated as proposed by $[24,25]$, that is, every $250 \mathrm{~ms}$, yielding 7200 samples for a recording of 30 minutes.

\section{Methods}

As previously reported, the foetal heart rate was estimated in real time from ultrasound Doppler signals $[24,25]$ and then recorded. The coarse-graining from HRV recordings procedure was performed offline. Then segmentation was applied. Scaling factors and multifractal spectra were subsequently evaluated using the structure function (see the scheme in Figure 2).

3.1. Coarse-Grained Analysis. Each time series $x(n)$ composed of $M=7200$ points was analysed from FHR recordings. Multiscale analysis was introduced to capture the fluctuations present in the time series at different scales. This method consisted of evaluating approximate versions of the original time series from a local average of neighbouring

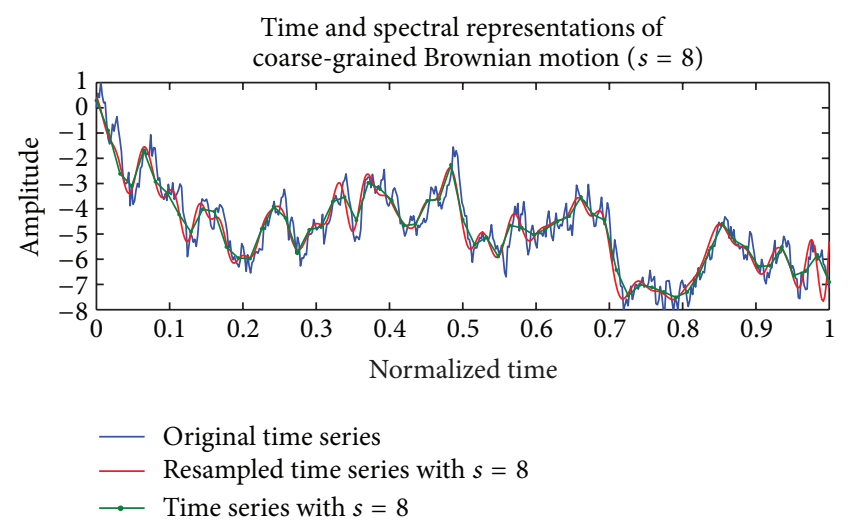

(a)

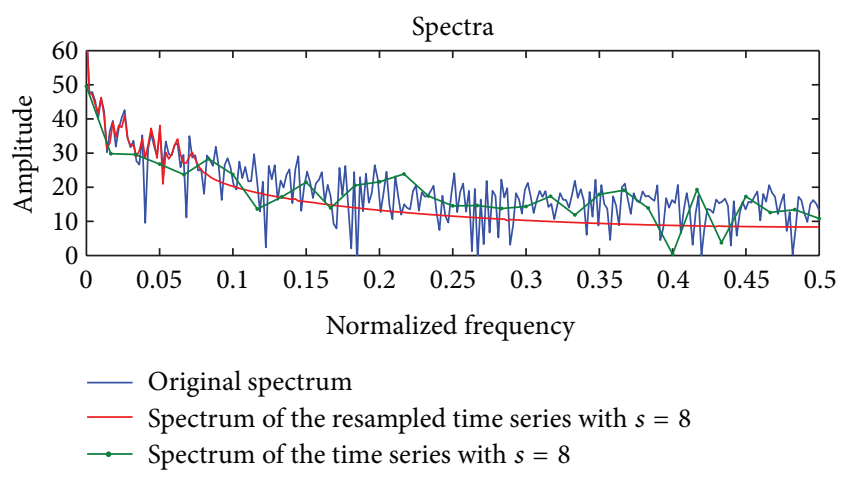

(b)

FIGURE 1: Time and spectral representations of a Brownian motion. (a) Original time series superimposed on the coarse-grained time series $(\alpha=8)$ and the resampled coarse-grained time series $(\alpha=8)$. (b) Spectrum of each time series depicted in (a).

points. This procedure is named "coarse-grained" [18]. The new reduced time series composed of $M / \alpha$ samples at scale $\alpha$ was written as

$$
y_{\alpha}(k)=\frac{1}{\alpha} \sum_{i=(k-1) \alpha+1}^{k \alpha} x_{i},
$$

for $1 \leq k \leq M / \alpha, y_{1}(k)=x(k)$ being the original time series. Figure 1 sets out the time and the spectral representations of coarse-grained Brownian motion time series used to calculate one of the effects resulting from the coarse-grained procedure. The time and the frequency were normalized. Figure 1(a) shows the original time series superimposed on the coarse-grained time series with $\alpha=8$ and the resampled coarse-grained time series with $\alpha=8$. Note that a resampled coarse-grained time series was an interpolated and filtered time series by a factor $\alpha$. The resampled coarse-grained times series was composed of $M$ samples.

Figures 1(a) and 1(b) show clearly that the coarse-grained time series were filtered time series. It can be claimed from these outcomes that the reducing duration of each coarsegrained times series is a side effect that can be avoided by resampling. In the following the multifractal descriptors were evaluated from resampled coarse-grained time series. 


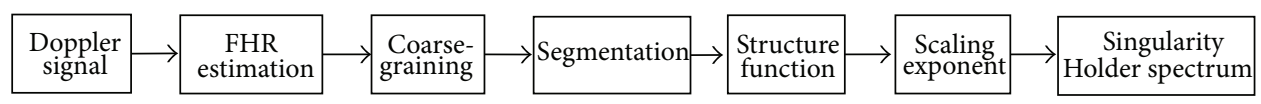

FIGURE 2: Scheme of different processes used to calculate coarse-grained multifractal descriptors.

As previously shown by [26], the more the fBm was filtered, the more the filtered $\mathrm{fBm}$ was regular: it can therefore be claimed that the higher the scale factor $\alpha$, the higher the Hurst exponent $H$. In the study by [27], it was shown that the coarse-graining affected the anticorrelated time series $(H<$ $0.5)$ in a more pronounced way than the correlated time series $(H>0.5)$. By supposing the coarse-grained effect to be a low pass filtering effect, we suggest that this can be understood in the following way.

(i) For $H<0.5$, fBm has several high frequency components that can be removed by the coarse-graining. Time series before and after filtering are quite different, indicating that the coarse-graining has a nonnegligible effect on time series.

(ii) For $H>0.5$, fBm has several low frequency components that are slightly removed by the coarse-graining effect. Time series before and after filtering were fairly similar, indicating that the coarse-graining has a negligible effect on time series.

Figure 3 represents different multifractal descriptors for different $\mathrm{fBm}$ of Hurst exponents $H=\{0.1,0.5,1\}$ with different scale factors $\alpha=\{1,3,6\}$. The fBm under consideration was composed of 720 samples. The results derived from Figure 3 showed that the anticorrelated $\mathrm{fBm}$ of Hurst exponent $H=0.1$ was more affected by the coarse-graining effect than the correlated $\mathrm{fBm}$ of Hurst exponent $H=1$. These results were compatible with those reported by $[26,27]$.

3.2. Multifractal Analysis. Due to the nonstationary nature of the coarse-grained time series analysed, a short-term procedure was performed. This procedure consisted of evaluating multifractal descriptors from subsignals $y_{\alpha}^{(i)}(k)$ composed of $N=720$ points $(3 \mathrm{~min})$.

Among all the existing methods supplying multifractal descriptors, we used the structure function of order $q$. Although it has been demonstrated theoretically that for certain types of signals the methods based on structure function of order $q$ have limitations for $q<0$, we believe that this type of approach is still worth using because of the following.

(i) The structure function is by far the simplest method to implement compared to DFA, box counting, and wavelet methods.

(ii) Using $q<0$ is valuable for analysing very small variations in time series. However, as time series were mostly corrupted by noise, it was impossible to probe small variations in the time series clearly. The practical value of such a negative order $q$ was strongly limited by the presence of noise. (iii) The real signals under consideration were not theoretical signals. This means that mathematical demonstrations operating exclusively on theoretical signals are not systematically applicable in practice.

(iv) Several multifractal analyses showed that it was more possible to discriminate between normal and distressed subjects for $q>0$ than for $q<0$. This was particularly the case in (i) [13] where it was shown that the difference between the scaling exponent $\tau(q)$ obtained for healthy and distressed subjects was greater for $q>0$ than for $q<0$ and in (ii) $[28,29]$ where it was clearly shown that for $q>0$ it was possible to discriminate patients better with atropine than with placebo.

The structure function that we used in this study is defined [23] for $q>0$ as follows:

$$
Q(q, \epsilon)=\left(\int\left|y_{\alpha}^{(i)}(t+\epsilon)-y_{\alpha}^{(i)}(t)\right|^{q} d t\right)^{1 / q} .
$$

This structure function is a length measurement [26] where the term $\left|y_{\alpha}^{(i)}(t+\epsilon)-y_{\alpha}^{(i)}(t)\right|^{q}$ reveals a local behaviour while the term $\left(\int \cdots d t\right)^{1 / q}$ reveals a global behaviour.

If $Q(q, \epsilon)=K \epsilon^{\eta(q)}$, then the scaling exponent $\eta(q)$ is expressed (demonstration: $\log Q / \log \epsilon=\eta-(\log K / \log \epsilon)$ and $\left.\lim _{\epsilon \rightarrow 0}(\log Q / \log \epsilon)=\eta\right)$ as

$$
\eta(q)=\lim _{\epsilon \rightarrow 0} \frac{\log (Q(q, \epsilon))}{\log (\epsilon)} .
$$

Note that for a fractional Brownian motion of Hurst exponent $H$, the scaling exponent is $\eta(q)=H$. From the previous equation, the singularity spectrum $D(q)$ can be evaluated as follows:

$$
D(q)=q^{2} \frac{d \eta(q)}{d q}+1
$$

The Holder spectrum is written as

$$
h(q)=q \frac{d \eta(q)}{d q}+\eta(q) .
$$

Note that this singularity spectrum $D(q)$ can be obtained through a Legendre transform from $\tau(q)$ :

$$
D(q)=q \frac{d \tau(q)}{d q}-\tau(q),
$$

where $\tau(q)$ is another scaling exponent defined by

$$
\tau(q)=q \eta(q)-1
$$




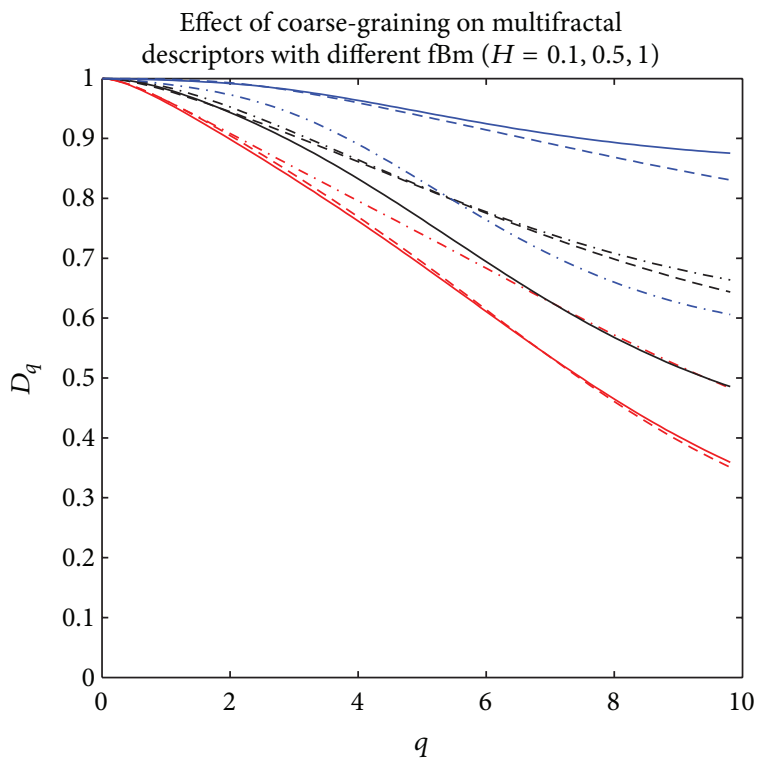

(a)

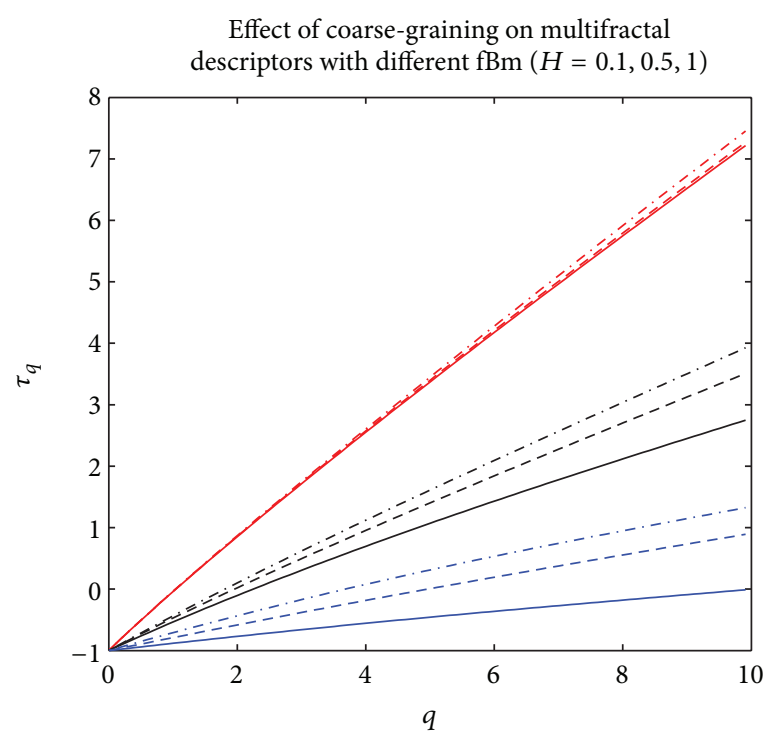

(c)

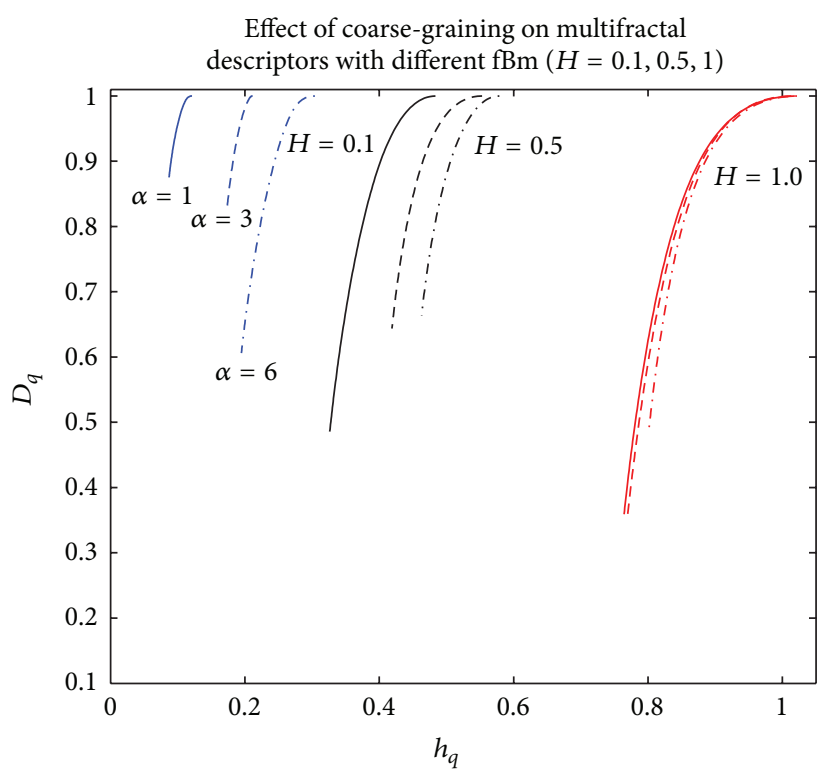

(b)

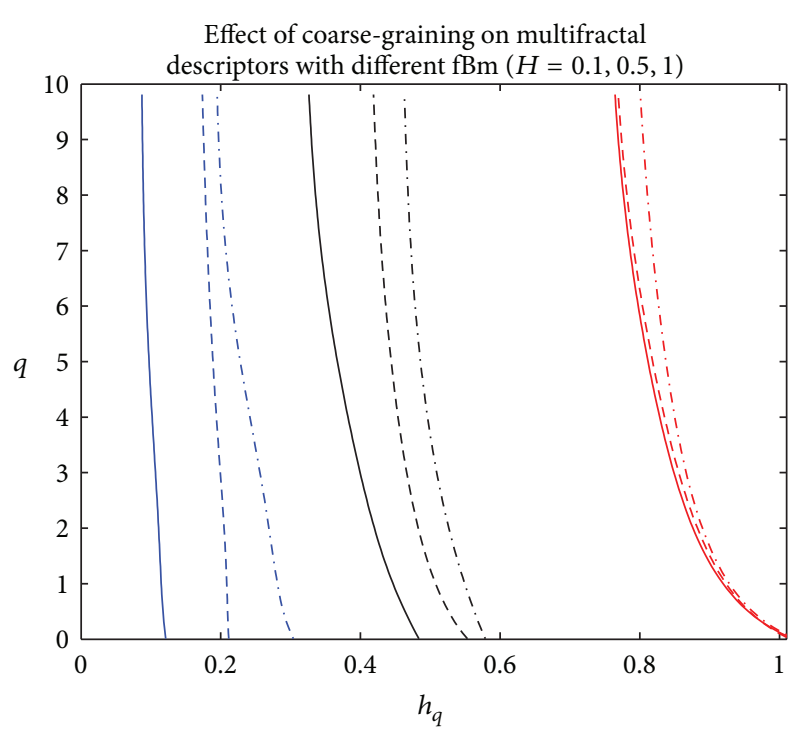

(d)

Figure 3: Effects of coarse-graining on multifractal descriptors for different $\mathrm{fBm}$ of Hurst exponents $H=\{0.1,0.5,1\}$ with different scale factors $\alpha=\{1,3,6\}$. (a) Singularity spectrum $D$ versus $q$. (b) Singularity spectrum $D$ versus Holder spectrum $h$. (c) Scaling exponent $\tau$ versus q. (d) Holder spectrum.

In this case, the Holder spectrum is written as

$$
h(q)=\frac{d \tau(q)}{d q}
$$

The structure function $Q(q, \epsilon)$ and the scaling exponents $\eta(q)$ and $\tau(q)$ for a normal foetus and a distressed foetus are reported in Figure 4 as an illustration. Figure 4(a) shows that the slopes of the curves obtained for different values of $q$ derived from the structure function $Q(d, \epsilon)$ were similar for the normal foetus. Similar results were derived for a distressed foetus. Figures 4(b) and 4(c) show that both scaling exponents $\eta(q)$ and $\tau(q)$ were more nonlinear for the healthy foetus than for the distressed foetus.

Other multifractal descriptors such as the singularity spectrum $D(q)$ and the Holder spectrum $h(q)$ are reported in Figure 5. The results set out in Figure 5 were obtained from four different signals: a signal from a distressed foetus of an estimated Hurst exponent $H=0.07$, a signal from a normal foetus of an estimated Hurst exponent $H=0.31$, and two fractional Brownian motion (fBm) signals of Hurst exponents $H=0.07$ and $H=0.31$. These four signals were each composed of 720 samples. Figure 5 shows that the magnitude of the dynamics of the singularity spectrum $D(q)$ and the 


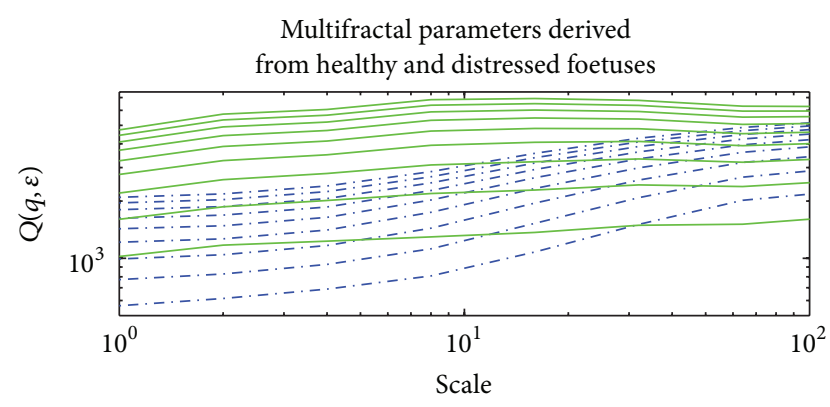

(a)

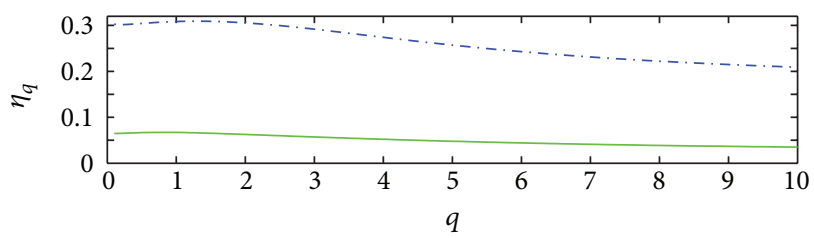

(b)

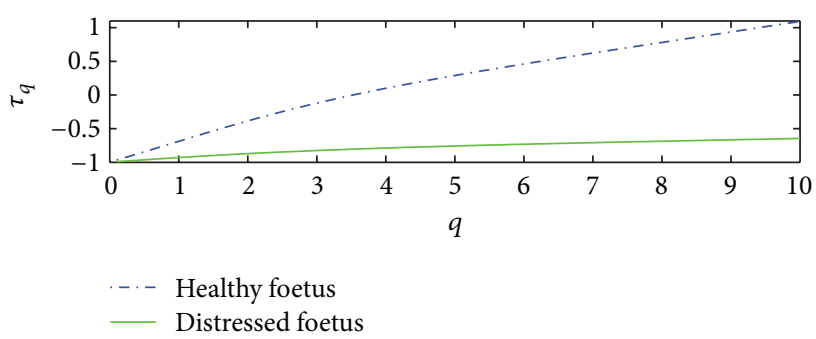

(c)

Figure 4: Multifractal parameters for a normal foetus (in blue) and a distressed foetus (in green). (a) Structure function $Q(q, \epsilon)$ versus scale. (b) Scaling exponent $\eta$ versus $q$. (c) Scaling exponent $\tau$ versus $q$. The two scaling exponents were more nonlinear for a healthy foetus than for a distressed foetus.

Holder spectrum $h(q)$ was higher for the healthy foetus than for the distressed foetus. Similarly, the magnitude of the dynamics of $D(q)$ and $h(q)$ was higher for foetal signals than for the $\mathrm{fBm}$ of the Hurst exponent, as for foetal signals. This corroborates most of the studies based on the analysis of multifractal HRV [12] where a more pronounced multifractal feature for healthy subjects was demonstrated than for distressed subjects. The Holder spectrum for healthy and distressed foetuses decreased with increasing values of $q$, thus supporting the multifractal nature of FHR time series. Such results are consistent with previous similar studies $[12,16]$. Note that normal and distressed fetal heart rate time series were reported in Figure 6 as an illustration.

Several measurements were performed in order to quantify the different trends observed in the multifractal indicators $D(q)$ and $h(q)$ for different scales $\alpha$.

(i) The relative error $\mathrm{RE}_{1}$ (in \%) of the Hurst exponent is defined as follows:

$$
\operatorname{RE}_{1}(\alpha)=\frac{\left|\bar{H}_{n}(\alpha)-\bar{H}_{d}(\alpha)\right|}{\bar{H}_{n}(\alpha)},
$$

where $\eta(q)=H$ is the Hurst exponent for all $q$. Note that $H=\eta(1) \cdot \bar{H}_{n}$ was the mean Hurst exponent corresponding to the average value obtained for all normal foetuses and $\bar{H}_{d}$ was the mean Hurst exponent corresponding to the average value obtained for all distressed foetuses.

(ii) The relative error $\mathrm{RE}_{2}$ (in \%) of the dynamics of $h(q)$ is defined as follows:

$$
\mathrm{RE}_{2}=\frac{\left|\overline{\Delta_{h n}}-\overline{\Delta_{h d}}\right|}{\overline{\Delta_{h n}}},
$$

where $\Delta_{h}=\max (h)-\min (h)$ are the dynamics of $h(q)$, $\overline{\Delta_{h n}}$ being the mean dynamics corresponding to the average value obtained for all normal foetuses and $\overline{\Delta_{h d}}$ the mean dynamics corresponding to the average value obtained for all distressed foetuses.

(iii) The relative error $\mathrm{RE}_{3}$ (in \%) is defined as follows:

$$
\mathrm{RE}_{3}=\frac{\left|\bar{D}_{n}-\bar{D}_{d}\right|}{\bar{D}_{n}},
$$

where $\bar{D}=$ mean $(D(q))$ is the mean value of the singularity spectrum, $\bar{D}_{n}$ being the mean value corresponding to the average value obtained for all normal foetuses and $\bar{D}_{d}$ the mean value corresponding to the average value obtained for all distressed foetuses.

(iv) The relative error $\mathrm{RE}_{4}$ (in \%) is defined as follows:

$$
\mathrm{RE}_{4}=\frac{\left|\overline{\Delta_{D n}}-\overline{\Delta_{D d}}\right|}{\overline{\Delta_{D n}}},
$$

where $\Delta_{D}=\max (D(q))-\min (D(q))$ is the mean value of the singularity spectrum, $\overline{\Delta_{D n}}$ being the mean value corresponding to the average value obtained for all normal foetuses and $\overline{\Delta_{D n}}$ the mean value corresponding to the average value obtained for all distressed foetuses.

\section{Results and Discussion}

From our own dataset composed of one hundred recordings, each time series of 7200 points was coarse-grained for 6 different scales. From each coarse-grained signal, subsignals composed of 720 points and overlapping by $97 \%$ were analysed with multifractal tools.

Figure 7 shows a boxplot representation of the mean Hurst exponent for different scale values ranging from 1 to 6 . Red boxplots correspond to distressed foetuses and blue boxplots correspond to normal foetuses. Figure 7 shows that the mean Hurst exponent for normal foetuses was higher than that obtained for distressed foetuses. This meant that the signatures of distressed foetuses were more irregular and complex than those obtained for normal foetuses. Furthermore, Figure 7 shows that there was sufficient deviation between the 


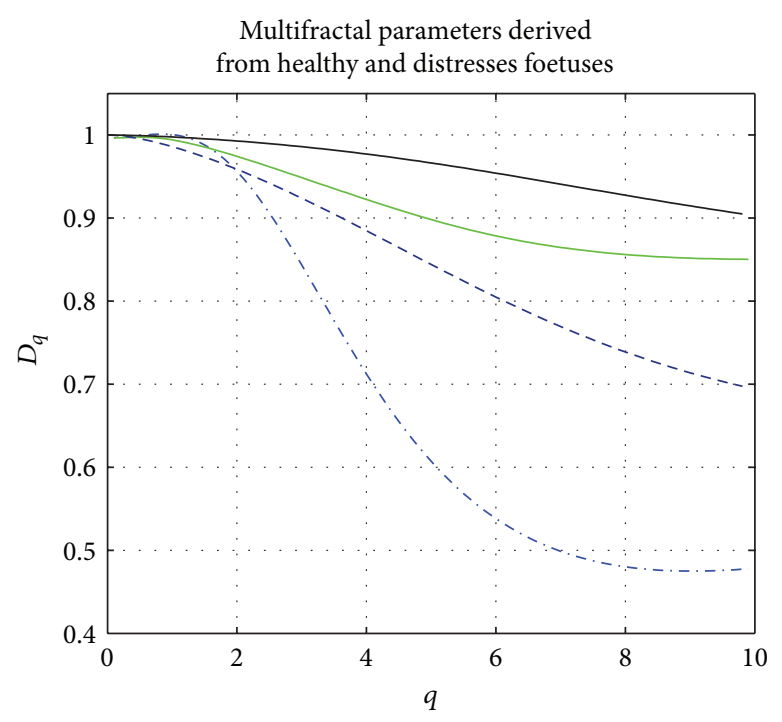

(a)

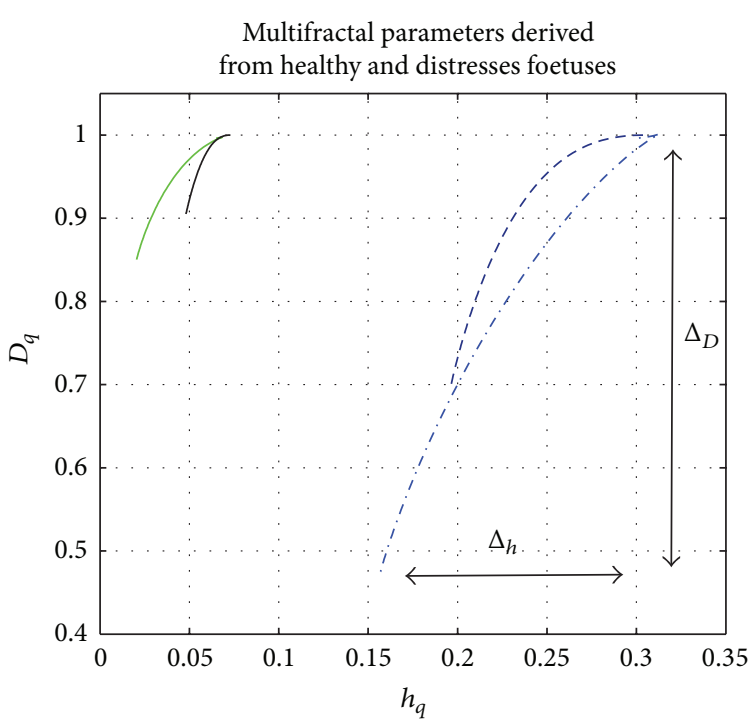

(b)

Multifractal parameters derived from healthy and distresses foetuses

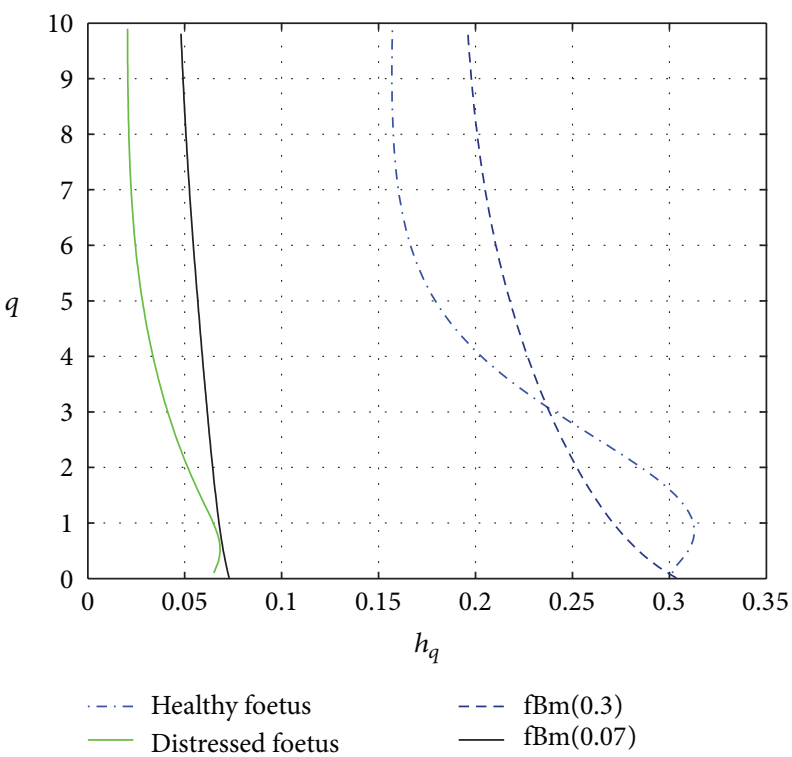

(c)

Figure 5: Multifractal parameters for a normal foetus (in blue) and a distressed foetus (in green). (a) Singularity spectrum $D$ versus $q$. (b) Singularity spectrum $D(q)$ versus Holder spectrum $h(q)$. (c) Holder spectrum $h$ versus $q$.

mean Hurst exponent to discriminate between normal and distressed foetuses. Figure 7 also shows that the higher the scale, the more regular or filtered the signal (as shown in Figure 1). This corroborated the results of [26], showing that the more filtered the time series the higher the Hurst exponent.

Figure 8 shows a boxplot representation of $\Delta_{h}=\max (h)-$ $\min (h)$. These dynamics are represented for different scale values from 1 to 6 . Red boxplots correspond to distressed foetuses and blue boxplots to normal foetuses. Figure 8 shows that the dynamics were higher for normal foetuses than that obtained for distressed foetuses. This meant that the signatures for normal foetuses were more multifractal than those obtained for distressed foetuses. This has already been reported in recent studies such as [12]. Furthermore, Figure 8 shows that there was sufficient deviation between the dynamics to distinguish normal from distressed foetuses.

Figure 9 shows a boxplot representation of the mean singularity spectrum $\bar{D}=D_{\text {mean }}$. This parameter was represented for different scale values ranging from 1 to 6 . Red boxplots correspond to distressed foetuses and blue boxplots to normal foetuses. Figure 9 shows that $\bar{D}$ was higher for distressed foetuses than for normal foetuses. This meant that the signatures of healthy foetuses were more regular than those 


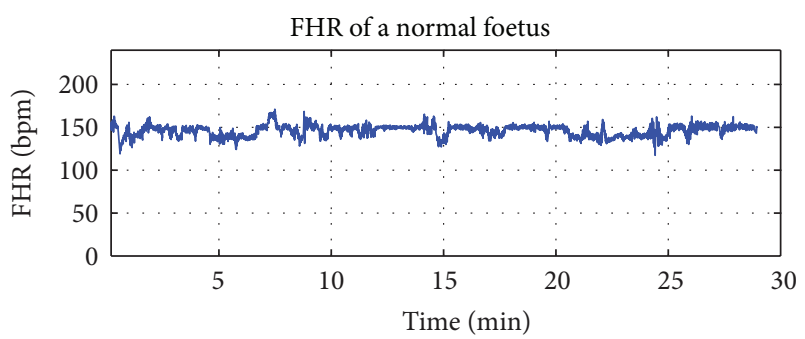

(a)

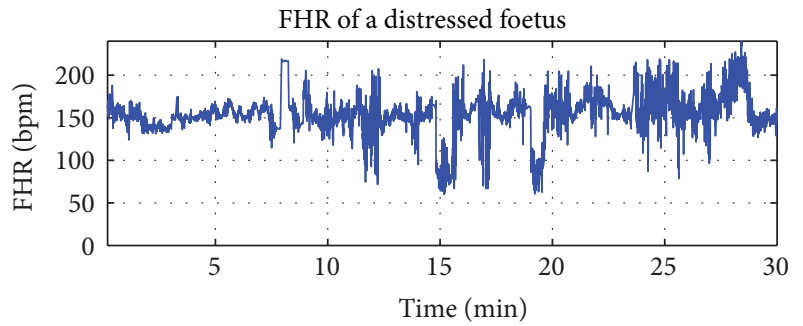

(b)

FIGURE 6: FHR of a normal foetus and a distressed foetus.

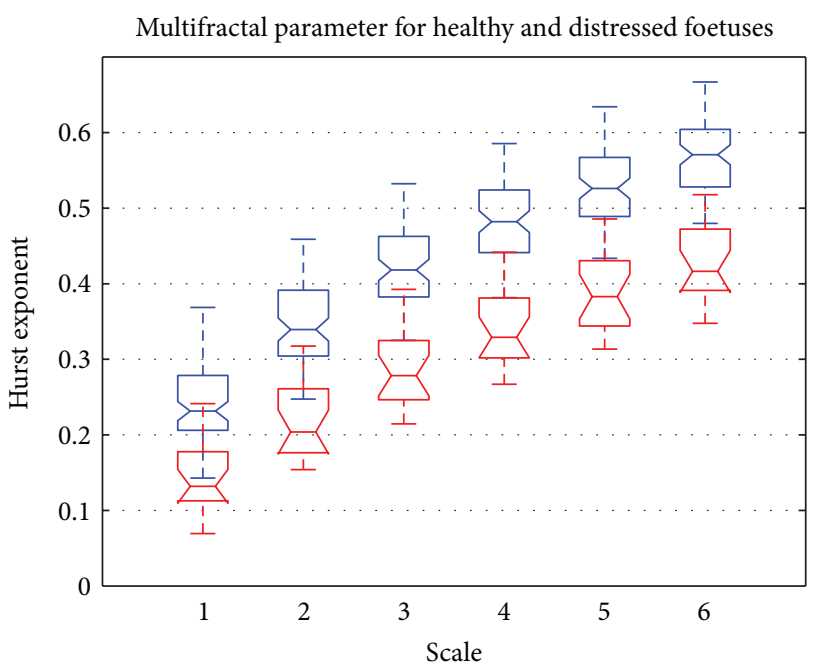

FIgURE 7: Boxplot of Hurst exponents versus scale. Normal foetus (in blue) and distressed foetus (in red).

corresponding to distressed foetuses. Figure 9 also shows that it was more difficult to discriminate between normal and distressed foetuses. This parameter did not seem to be very relevant. Note also that the higher the scale, the lower the relative error.

Figure 10 shows a boxplot representation of $\Delta_{D}$, that is, the dynamics of $D$. This dynamics is represented for different scale values from 1 to 6 . Red boxplots correspond to distressed foetuses and blue boxplots to normal foetuses. Figure 10 shows that $\Delta_{D}$ was higher for normal foetuses than for distressed foetuses. This meant that the signatures of healthy foetuses were more multifractal than those for distressed foetuses. Figure 10 also shows that there was sufficient deviation between the dynamics to distinguish normal from distressed

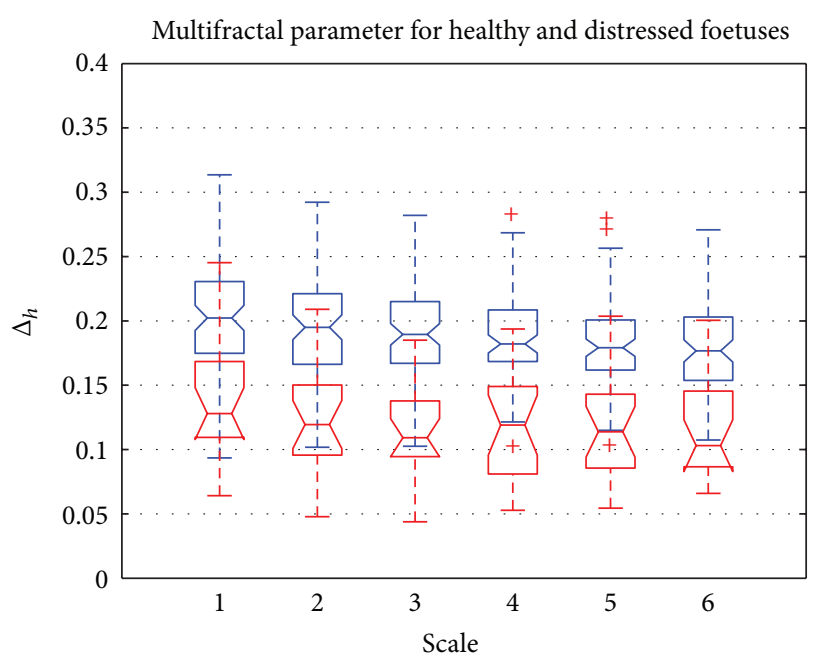

FIGURE 8: Boxplot of $\Delta_{h}=\max (h)-\min (h)$ versus scale. Normal foetus (in blue) and distressed foetus (in red).

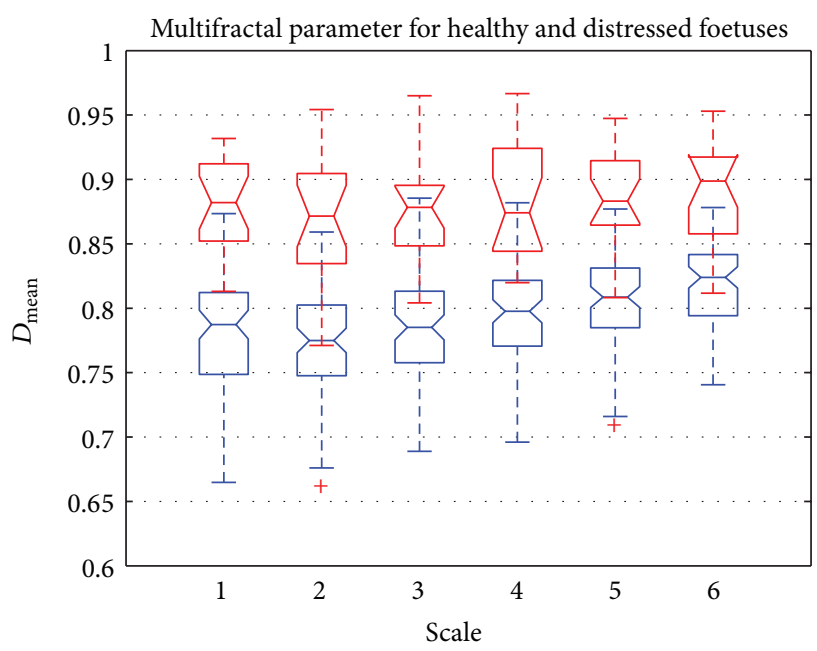

FIgURE 9: Boxplot of $D_{\text {mean }}$ versus scale. Normal foetus (in blue) and distressed foetus (in red).

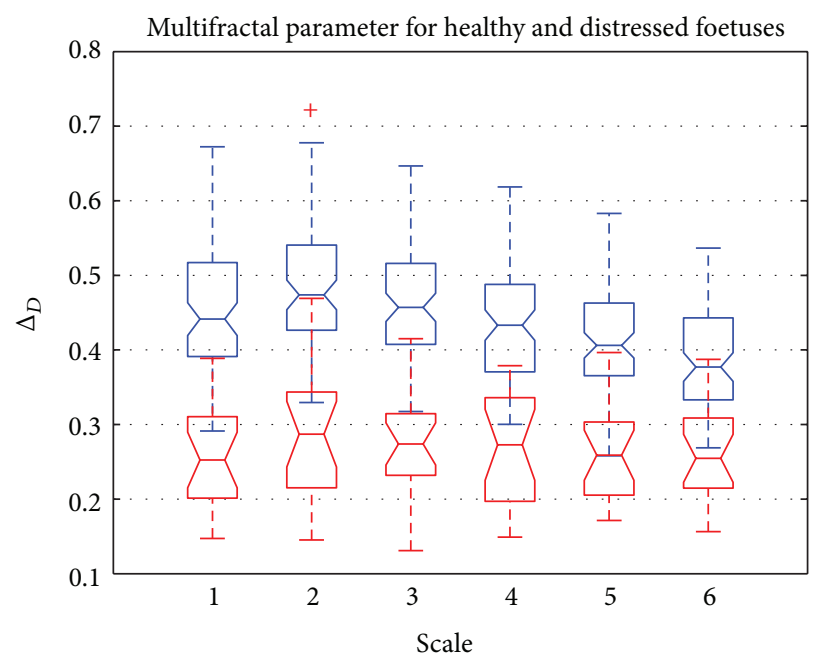

Figure 10: Boxplot of $\Delta_{D}$ versus scale. Normal foetus (in blue) and distressed foetus (in red). 
TABLE 1: Relative errors of different multifractal parameters between the two groups of foetuses for different scales.

\begin{tabular}{lcccccc}
\hline Scale & 1 & 2 & 3 & 4 & 5 & 6 \\
\hline $\mathrm{RE}_{1}$ & 0.37 & $\mathbf{0 . 4 0}$ & 0.33 & 0.29 & 0.26 & 0.24 \\
$\mathrm{RE}_{2}$ & 0.32 & $\mathbf{0 . 3 7}$ & $\mathbf{0 . 3 8}$ & 0.37 & 0.36 & 0.35 \\
$\mathrm{RE}_{3}$ & 0.11 & $\mathbf{0 . 1 2}$ & 0.11 & 0.10 & 0.09 & 0.08 \\
$\mathrm{RE}_{4}$ & 0.41 & $\mathbf{0 . 4 2}$ & 0.41 & 0.38 & 0.36 & 0.33 \\
\hline
\end{tabular}

foetuses. Note also that the higher the scale, the lower the relative error.

To conclude, Table 1 summarizes the relative errors of the four previous parameters. The findings derived from Table 1 showed that the best parameter permitting discrimination between foetuses was $\mathrm{RE}_{4}$, followed by $\mathrm{RE}_{1}$ and $\mathrm{RE}_{2}$. Indeed the best differentiation was obtained for a scale value of 2 for $\mathrm{RE}_{4}$ and $\mathrm{RE}_{1}$ and a scale value of 3 for $\mathrm{RE}_{2}$. This confirms the need to coarse-grain the FHR time series. It is obvious from Table 1 that the higher the scale, the lower the relative error. This requires choosing a maximum scale that is not too high: a value set at 2 seems sufficient whatever the relative error. Furthermore, as the best parameter $\mathrm{RE}_{4}$ was sensitive to the multifractal features of the time series analyzed for a scale of 2 , this finding confirms the need to analyze FHR from a coarse-grained multifractal point of view. However, note also that as the second discriminative parameter was $\mathrm{RE}_{1}$, sensitive to monofractal features set at a scale of 2 , then a coarsegrained monofractal approach is also relevant.

Finally, although the present study was quite similar to that presented in [15], our study was different in several ways. First, our study was dedicated to foetuses, whereas [15] was dedicated to adults. Second, our study was based on a much simpler structure function than the other approach that was based on a partition function.

Furthermore, although a large number of research studies have mainly been based on the use of partition functions (DFA, box-counting and wavelet approaches) on the pretext that structure functions do not operate for negative orders, we have shown here (i) that the use of such structure functions is fully justified due to the simplicity of implementation and (ii) that structure functions completely fulfil their role in distinguishing between healthy and distressed foetuses.

Note that, as our proposed methodology was that of investigating offline, we plan to evaluate multifractal descriptors one line in the near future.

\section{Acknowledgments}

This study was supported by the "Agence Nationale de la Recherche" (Project ANR-07-TECSAN-023, Surfoetus). Furthermore, the authors would like to thank the Clinical Investigation Centre for Innovative Technology of Tours (CIC-IT 806 CHRU of Tours) and Professor F. Perrotin's team in the Obstetric Department for their support in recording the signals.

\section{References}

[1] D. Brodsky and H. Christou, "Current concepts in intrauterine growth restriction," Journal of Intensive Care Medicine, vol. 19, no. 6, pp. 307-319, 2004.

[2] M. Kobayashi and T. Musha, "1/f fluctuation of heartbeat period," IEEE Transactions on Biomedical Engineering, vol. 29, no. 6, pp. 456-457, 1982.

[3] M. Ferrario, M. Signorini, G. Magenes, and S. Cerutti, "Comparison of entropy-based regularity estimators: application to the fetal heart rate signal for the identification of fetal distress," IEEE Transactions on Biomedical Engineering, vol. 53, no. 1, pp. 119-125, 2006.

[4] M. Ferrario, M. Signorini, and G. Magenes, "Complexity analysis of the fetal heart rate variability: early identification of severe intrauterine growth-restricted fetuses," Medical and Biological Engineering and Computing, vol. 47, no. 9, pp. 911-919, 2009.

[5] S. M. Pincus and R. R. Viscallero, "Approximate entropy: a regularity measure for fetal heart rateanalysis," Obstetrics and Gynecology, vol. 79, pp. 249-255, 1992.

[6] H. Goncalves, J. Bernades, A. P. Rocha, and D. A. de Campos, "Linear and nonlinear analysis of heart rate patterns associated with fetal behavioral states in the antepartum period," Early Human Development, vol. 83, no. 9, pp. 585-591, 2007.

[7] D. G. Chaffin, C. C. Goldberg, and K. L. Reed, "The dimension of chaos in the fetal heart rate," American Journal of Obstetrics and Gynecology, vol. 165, no. 5, pp. 1425-1429, 1991.

[8] N. A. J. Gough, "Fractal analysis of fetal heart rate variability," Physiological Measurement, vol. 14, no. 3, pp. 309-315, 1993.

[9] C. S. Felgueiras, J. P. Marques de Sa, J. Bernardes, and S. Gama, "Classification of foetal heart rate sequences based on fractal features," Medical and Biological Engineering and Computing, vol. 36, no. 2, pp. 197-201, 1998.

[10] A. Kikuchi, N. Unno, T. Horikoshi, T. Shimizu, S. Kozuma, and Y. Taketani, "Changes in fractal features of fetal heart rate during pregnancy," Early Human Development, vol. 81, no. 8, pp. 655661, 2005.

[11] P. C. Ivanov, M. G. Rosenblum, C.-K. Peng et al., "Scaling behaviour of heartbeat intervals obtained by wavelet-based time- series analysis," Nature, vol. 383, no. 6598, pp. 323-327, 1996.

[12] P. C. Ivanov, L. A. N. Amaral, A. L. Goldberger et al., "Multifractality in human heartbeat dynamics," Nature, vol. 399, pp. 461465, 1999.

[13] P. C. Ivanov, L. A. N. Amaral, A. L. Goldberger et al., "From 1/f noise to multifractal cascades in heartbeat dynamics," Chaos, vol. 11, no. 3, pp. 641-652, 2001.

[14] J. Wang, X. Ning, and Y. Chen, "Modulation of heart disease information to the 12-lead ECG multifractal distribution," Physica A, vol. 325, no. 3-4, pp. 485-491, 2003.

[15] J. Wang, X. Ning, Q. Ma, C. Bian, Y. Xu, and Y. Chen, "Multiscale multifractality 260 analysis of a 12-lead electrocardiogram," Physical Review E, vol. 71, no. 6, Article ID 062902, 4 pages, 2005.

[16] R. Sassi, M. Signorini, and S. Cerutti, "Multifractality and heart rate variability," Chaos, vol. 19, no. 2, Article ID 028507, 2009.

[17] A. Humeau, B. Buard, G. Mahé, F. Chapeau-Blondeau, D. Rousseau, and P. Abraham, "Multifractal analysis of heart rate variability and laser Doppler flowmetry fluctuations: comparison of results from different numerical methods," Physics in Medicine and Biology, vol. 55, no. 20, pp. 6279-6297, 2010. 
[18] Y. C. Zhang, "Complexity and $1 / f$ noise: a phase space approach," Journal de Physique Archives, vol. 1, no. 7, pp. 971-977, 1991.

[19] A. L. Barabasi and T. Vicsek, "Multifractality of self-affine fractals," Physical Review A, vol. 44, no. 4, pp. 2730-2733, 1991.

[20] J. F. Muzy, E. Bacry, and A. Arneodo, "Multifractal formalism for fractal signals: the structure-function approach versus the wavelet-transform modulus-maxima method," Physical Review E, vol. 47, no. 2, pp. 875-884, 1993.

[21] D. C. Lin and R. L. Hughson, "Modeling heart rate variability in healthy humans: a turbulence analogy," Physical Review Letters, vol. 86, no. 8, pp. 1650-1653, 2001.

[22] E. S. C. Ching and Y.-K. Tsang, "Multifractality and scale invariance in human heartbeat dynamics," Physical Review E, vol. 76, no. 4, Article ID 041910, 2007.

[23] G. Parisi and U. Frisch, "On the singularity structure of fully developed turbulence (in turbulence and predictability in geophysical fluid dynamics)," in Proceedings of the International School of Physics Enrico Fermi, M. Ghil, R. Benzi, and G. Paris, Eds., pp. 84-87, North-Holland .

[24] I. Voicu, J. M. Girault, and S. Menigot, "Improved estimation of the fetal heart rate using directional Doppler signal and YIN," IRBM, vol. 33, no. 4, pp. 262-271, 2012.

[25] I. Voicu, S. Menigot, D. Kouamé, and J. M. Girault, "New estimators and guidelines for better use of fetal heart 14 rate estimators with Doppler ultrasound devices," Computational and Mathematical Methods in Medicine, vol. 2013, Article ID 784862, 2013.

[26] J.-M. Girault, D. Kouame, and A. Ouahabi, "Analytical formulation of the fractal dimension of filtered stochastic signals," Signal Processing, vol. 90, no. 9, pp. 2690-2697, 2010.

[27] Y. Xu, Q. D. Y. Ma, D. T. Schmitt, P. Bernaola-Galvan, and P. C. Ivanov, "Effects of coarse-graining on the scaling behavior of long-range correlated and anti-correlated signals," Physica A, vol. 390, no. 23-24, pp. 4057-4072, 2011.

[28] H. E. Stanley, L. A. N. Amaral, A. L. Goldberger, S. Havlin, P. C. Ivanov, and C.-K. Peng, "Statistical physics and physiology: monofractal and multifractal approaches," Physica A, vol. 270, no. 1, pp. 309-324, 1999.

[29] L. A. N. Amaral, P. C. Ivanov, N. Aoyagi et al., "Behavioral-independent features of complex heartbeat dynamics," Physical Review Letters, vol. 86, no. 26, pp. 6026-6029, 2001. 


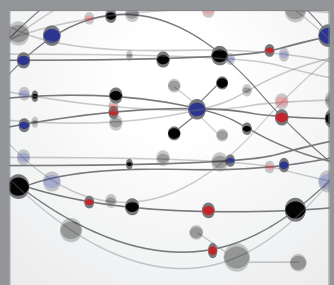

The Scientific World Journal
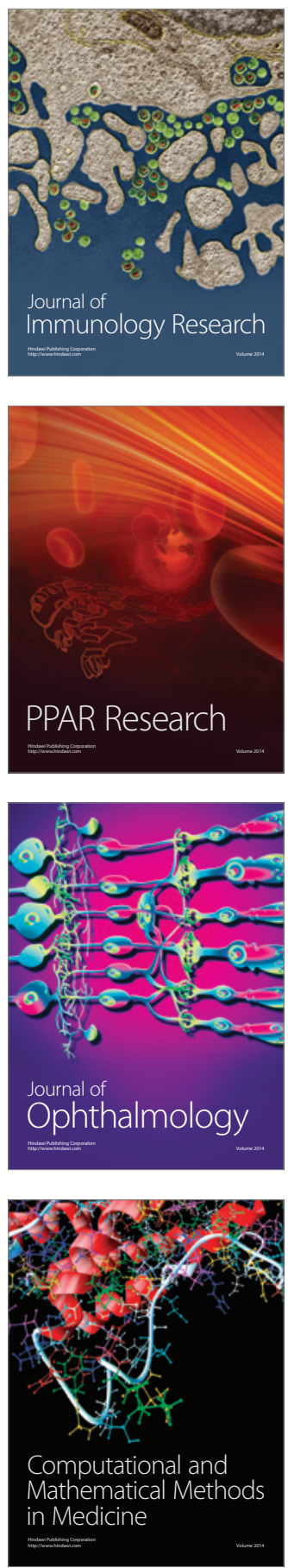

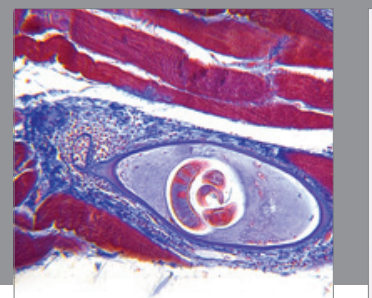

Gastroenterology

Research and Practice
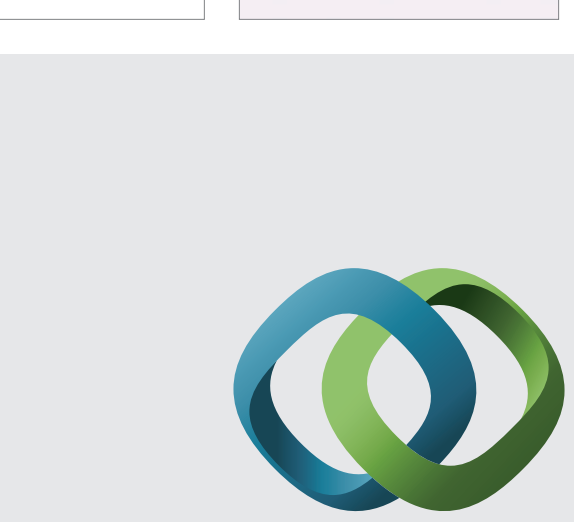

\section{Hindawi}

Submit your manuscripts at

http://www.hindawi.com
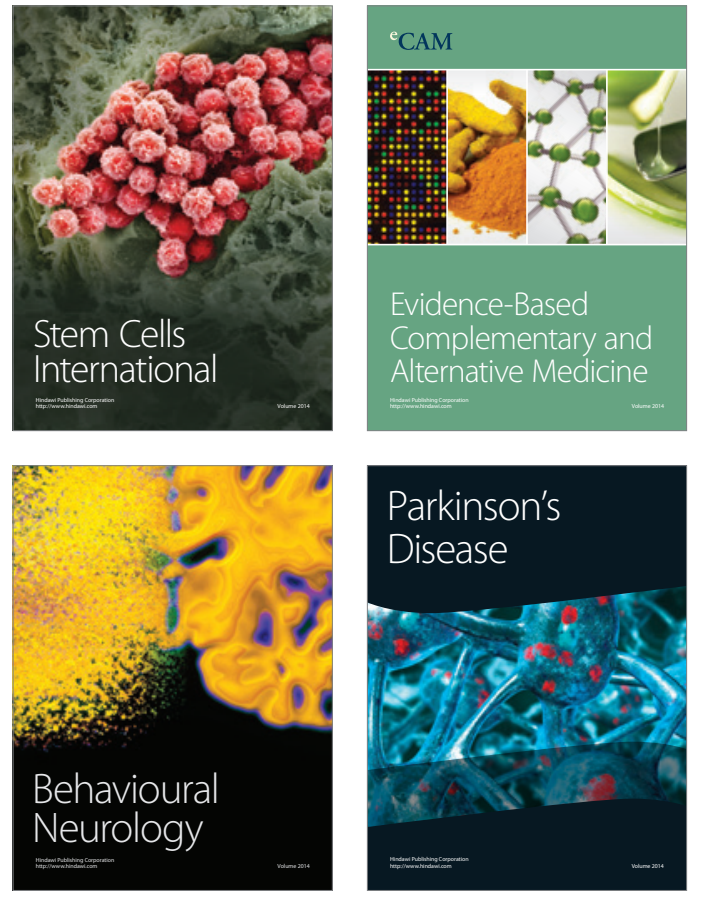
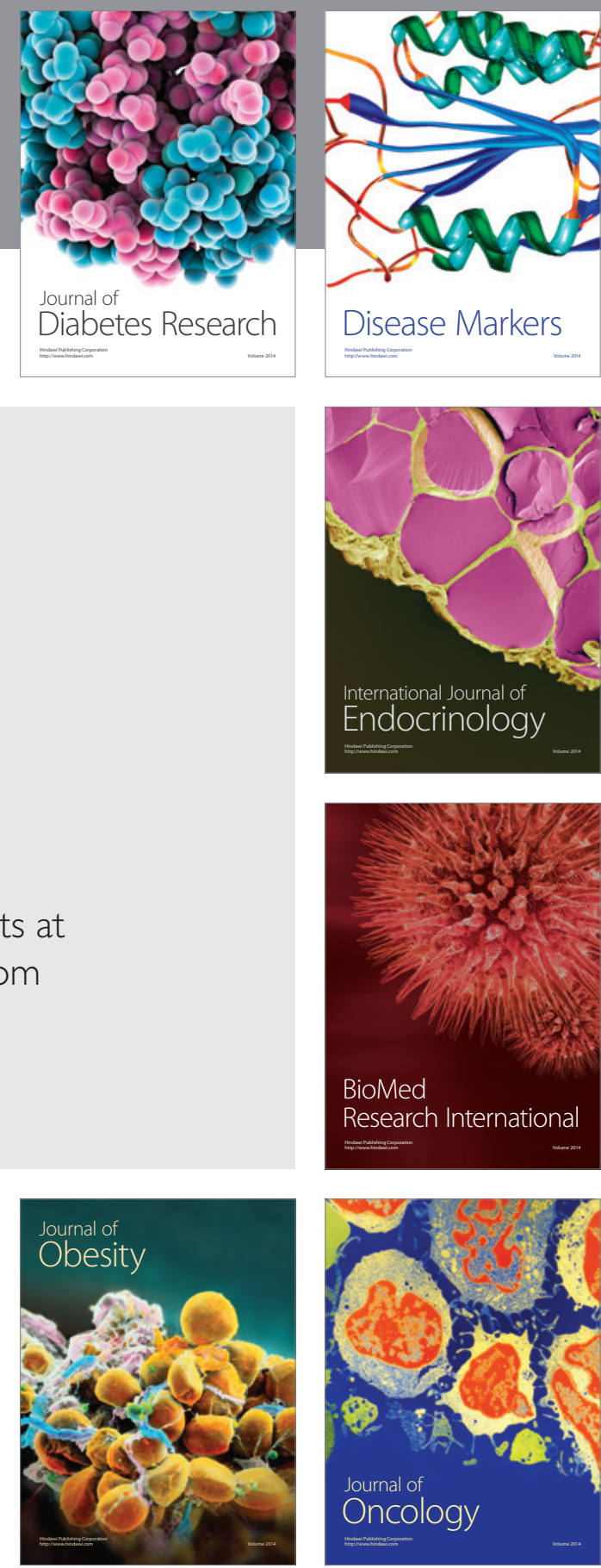

Disease Markers
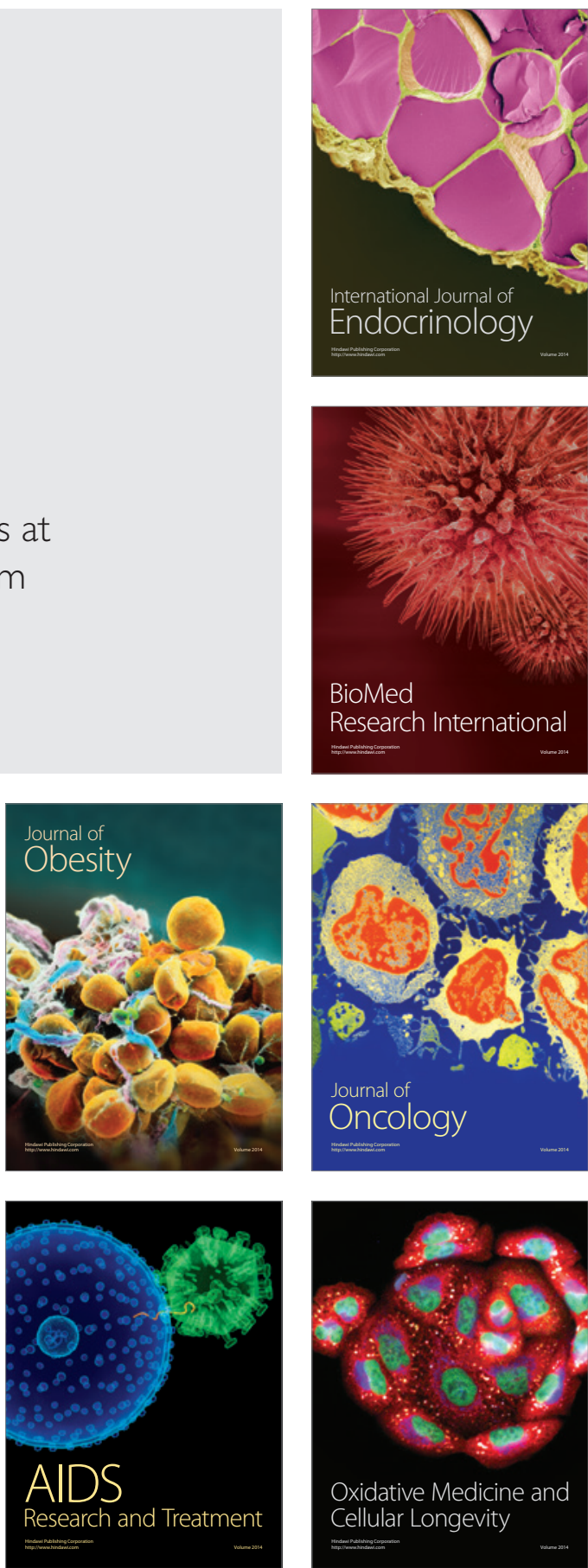